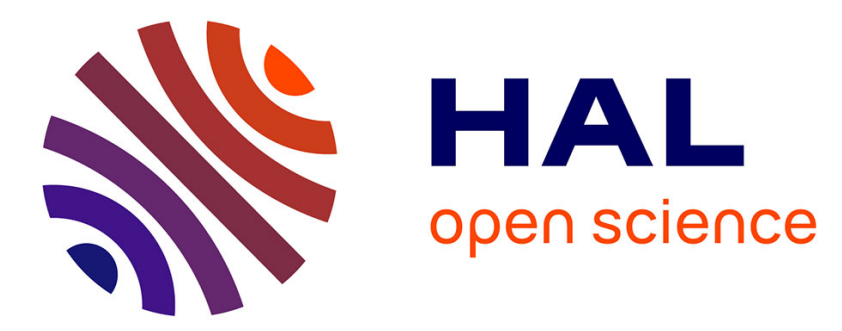

\title{
Bootstrapping heteroskedasticity consistent covariance matrix estimator
}

\author{
Emmanuel Flachaire
}

\section{To cite this version:}

Emmanuel Flachaire. Bootstrapping heteroskedasticity consistent covariance matrix estimator. Computational Statistics, 2002, 17 (4), pp.501-506. halshs-00175897

\section{HAL Id: halshs-00175897 \\ https://shs.hal.science/halshs-00175897}

Submitted on 1 Oct 2007

HAL is a multi-disciplinary open access archive for the deposit and dissemination of scientific research documents, whether they are published or not. The documents may come from teaching and research institutions in France or abroad, or from public or private research centers.
L'archive ouverte pluridisciplinaire HAL, est destinée au dépôt et à la diffusion de documents scientifiques de niveau recherche, publiés ou non, émanant des établissements d'enseignement et de recherche français ou étrangers, des laboratoires publics ou privés. 


\title{
Bootstrapping Heteroskedasticity \\ Consistent Covariance Matrix Estimator
}

\author{
by \\ Emmanuel Flachaire \\ EurequA, University Paris I Panthéon-Sorbonne
}

December 2001

\begin{abstract}
Recent results of Cribari-Neto and Zarkos (1999) show that bootstrap methods can be successfully used to estimate a heteroskedasticity robust covariance matrix estimator. In this paper, we show that the wild bootstrap estimator can be calculated directly, without simulations, as it is just a more traditional estimator. Their experimental results seem to conflict with those of MacKinnon and White (1985); we reconcile these two results.
\end{abstract}

JEL classification: C1

Keywords: wild bootstrap, heteroskedasticity 


\section{Introduction}

In this paper, we show that the wild bootstrap covariance matrix estimator can be calculated directly, without simulation, since it is simply a traditional heteroskedasticity consistent covariance matrix estimator first proposed by Eicker (1963) and White (1980), see section 2. Simulation results in MacKinnon and White (1985) had suggested that the jackknife is a reliable heteroskedasticity-consistent variance estimator. Simulation results in Cribari-Neto and Zarkos (1999) suggest that this estimator did not perform as well as its competitors. This conclusion appears to show a conflict between different simulation results and could lead us to the wrong conclusion that the jackknife estimator is not reliable. In section 3 , we reconcile these experimental results.

\section{HCCME and bootstrap estimators}

Consider the linear heteroskedastic model:

$$
y_{t}=X_{t} \beta+u_{t} \quad E\left(u_{t} \mid X\right)=0, \quad E\left(u_{t}^{2} \mid X\right)=\sigma_{t}^{2}
$$

where $y_{t}$ is a dependent variable, $X_{t}$ a regressor row matrix $1 \times k, \beta$ and $\sigma_{t}$ are respectively the unknown parameters and the unknown variances of the error terms; $u_{t}$ is error term. We consider only unconditional heteroskedasticity, which means that the $\sigma_{t}^{2}$ may depend on the exogeneous regressors, but not, for instance, on lagged dependent variables. The Heteroskedasticity Consistent Covariance Matrix Estimator, or HCCME, is

$$
\left(X^{\top} X\right)^{-1} X^{\top} \hat{\Omega} X\left(X^{\top} X\right)^{-1}
$$

where the $n \times n$ diagonal matrix $\hat{\Omega}$ has typical diagonal element $a_{t}^{2} \hat{u}_{t}^{2}$, where $\hat{u}_{t}$ is the OLS residual. We refer to the basic version of the HCCME, proposed by Eicker (1963) and White (1980), as $H C_{0}$ where $a_{t}=1$. Bias is reduced by multiplying the $\hat{u}_{t}$ by the square root of $n /(n-k)$, thereby by multiplying the elements of $\hat{\Omega}$ by $n /(n-k)$; this procedure, analogous to the use in the homoskedastic case of the unbiased OLS estimator of the error variance, gives rise to form $H C_{1}$ of the HCCME where $a_{t}=(n /(n-k))^{1 / 2}$. In the homoskedastic case, the variance of $\hat{u}_{t}$ is proportional to $1-h_{t}$, where $h_{t} \equiv X_{t}\left(X^{\top} X\right)^{-1} X_{t}^{\top}$, the $t^{\text {th }}$ element of the orthogonal projection matrix on to the span of the columns of $X$. This suggests $a_{t}=\left(1-h_{t}\right)^{-1 / 2}$ in order to obtain $\hat{\Omega}$. If this is done, we obtain form $H C_{2}$ of the HCCME. Finally, arguments based on the jackknife lead MacKinnon and White to propose form $H C_{3}$, for which $a_{t}=\left(1-h_{t}\right)^{-1}$. MacKinnon and White (1985), and Chesher and Jewitt (1987), show that, in terms of size distortion, $H C_{0}$ is outperformed by $H C_{1}$, which is in turn outperformed by $\mathrm{HC}_{2}$ and $\mathrm{HC}_{3}$. The last two cannot be ranked in general, although $\mathrm{HC}_{3}$ has been shown in a number of Monte carlo experiments to be superior in typical cases.

Heteroskedasticity of unknown form cannot be mimicked in the bootstrap distribution. The wild bootstrap gets round this problem by using a bootstrap data generating process, or bootstrap DGP, of the form

$$
y_{t}^{\star}=X_{t} \hat{\beta}+a_{t} \hat{u}_{t} \varepsilon_{t}^{\star}
$$


where $\hat{\beta}$ is a vector of parameter estimates, $a_{t}$ takes the forms $H C_{0}, H C_{1}, H C_{2}$ or $H C_{3}$ and the $\varepsilon_{t}^{\star}$ are mutually independent drawings, completely independent of the original data, from some auxiliary distribution such that $E\left(\varepsilon_{t}^{\star}\right)=0$ and $E\left(\varepsilon_{t}^{\star 2}\right)=1$. The heteroskedasticity consistent covariance matrix estimate of the OLS parameter estimate under the wild bootstrap DGP (3) is the wild bootstrap covariance matrix estimator. Cribari-Neto and Zarkos (1999) suggest to approximate the wild bootstrap estimator by the following steps:

1. Estimate the model (1) by Ordinary Least Squares (OLS): $\hat{\beta}$ and $\hat{u}_{t}$ are respectively the parameter estimates and the residuals.

2. Generate a bootstrap sample, based on the bootstrap data generating process: $y_{t}^{\star}=$ $X_{t} \hat{\beta}+a_{t} \hat{u}_{t} \varepsilon_{t}^{\star}$, where $\varepsilon_{t}^{\star}$ is drawn from a probability distribution $F$ with zero expectation and variance equal to one. Compute the OLS bootstrap parameter estimate $\hat{\beta}^{\star}=$ $\left(X^{\top} X\right)^{-1} X^{\top} y^{\star}$. Repeat this step $B$ times.

3. The variance of the $B$ bootstrap estimates is an approximation of the wild bootstrap covariance matrix estimate of $\hat{\beta}$.

We can now give the principal result of this section.

Theorem 1 For a fixed number of observations and under the data generating process (3), the wild bootstrap covariance matrix estimator is equal to the Heteroskedasticity Consistent Covariance Matrix Estimator (2).

Proof: The wild bootstrap data generating process is $y_{t}^{\star}=X_{t} \hat{\beta}+a_{t} \hat{u}_{t} \varepsilon_{t}^{\star}$. For a fixed number of observations $n$, the error terms are independent: $E\left(\varepsilon_{t}^{\star} \varepsilon_{s}^{\star} \mid X\right)=0$ with $t \neq s$. The ordinary least squares parameter estimate is equal to: $\hat{\beta}^{\star}=\left(X^{\top} X\right)^{-1} X^{\top} y^{\star}$ and its covariance matrix is

$$
\left(X^{\top} X\right)^{-1} X^{\top} \Omega^{\star} X\left(X^{\top} X\right)^{-1}
$$

where $E\left(u^{\star} u^{\star \top} \mid X\right)=\Omega^{\star}$ and $u^{\star}=a_{t} \hat{u}_{t} \varepsilon_{t}^{\star}$. The off-diagonal element of $\Omega^{\star}$ for row $t$ and column $s$, where $t \neq s$, is equal to:

$$
\Omega_{t, s}^{\star}=E\left(u_{t}^{\star} u_{s}^{\star} \mid X\right)=a_{t} \hat{u}_{t} a_{s} \hat{u}_{s} E\left(\varepsilon_{t}^{\star} \varepsilon_{s}^{\star} \mid X\right)=0
$$

The diagonal element $t$ is equal to:

$$
\Omega_{t, t}^{\star}=E\left(u_{t}^{\star 2} \mid X\right)=a_{t}^{2} \hat{u}_{t}^{2} E\left(\varepsilon_{t}^{2} \mid X\right)=a_{t}^{2} \hat{u}_{t}^{2}
$$

It is clear that, for a fixed number $n$ of observations, (4) is equal to (2).

This theorem implies that the wild bootstrap covariance matrix estimator is equal to the HCCME proposed by Eicker (1963), White (1980) and MacKinnon and White (1985). If we approximate the wild bootstrap covariance matrix estimator by simulations, we can observe numerical differences because a finite number $B$ of simulations introduces an experimental error. Consequently, the HCCME estimator should always be preferred to the wild bootstrap estimator obtained by simulations. 


\section{Experimental results}

Experimental results of Cribari-Neto and Zarkos (1999) show that the bias of the $\mathrm{HC}_{2}$ form of the wild bootstrap estimator is less than that of the jackknife estimator. These results are for the bias of the variance estimator, but MacKinnon and White (1985) results show that tests based on the jackknife estimator of the variance tend to be accurate, its bias notwithstanding. The two sets of results concern fundamentally different objects. In this section we directly compare the size and the bias of different tests. The results lead us to reconcile the conflict claimed by Cribari-Neto and Zarkos. In our Monte-Carlo experiments, we consider a simple linear regression model:

$$
y_{t}=\alpha+x_{t} \beta+u_{t} \quad u_{t} \sim N(0,1)
$$

The true parameters are: $\alpha_{0}=1$ and $\beta_{0}=0$, the error terms are independent and identically distributed following the standard Normal distribution. The sample size is small, $n=20$, and the regressor is supposed fixed ${ }^{1}$.

In subsection 3.1, we experimentally check the theoretical results obtained in the preceding section. In subsection 3.2, we study the bias of the estimators, as in Cribari-Neto and Zarkos (1999). In 3.3, we study the size distortion of the $t$-tests based on these estimators, as in MacKinnon and White (1985).

\subsection{Bootstrap and HCCME estimators}

We check the theoretical results of the preceding section: for a fixed number of observations $n$, the wild bootstrap bootstrap estimator converges to the HCCME estimator when the number of bootstrap samples $B$ increases to infinity. In our experiment, we draw one sample $(y, x)$ using (7), we calculate the HCCME estimator and the wild bootstrap estimator of $\hat{\beta}$ with $a_{t}=\left(1-h_{t}\right)^{-1 / 2}\left(H C_{2}\right.$ form) and we increase the number of bootstrap samples: $B=199 ; 499 ; 999 ; 9,999 ; 999,999$. We calculate two wild bootstrap estimators: one with the resampling distribution $F_{1} \sim N(0,1)$, and the other with $F_{2}$, a bi-atomic distribution yielding the value $-(\sqrt{5}-1) / 2$ with probability $p=(\sqrt{5}+1) / 2 \sqrt{5}$, and $(\sqrt{5}+1) / 2$ with probability $1-p$, see Liu (1988).

\begin{tabular}{|c||ccccc|}
\hline Design & $\mathrm{B}=199$ & $\mathrm{~B}=499$ & $\mathrm{~B}=999$ & $\mathrm{~B}=9,999$ & $\mathrm{~B}=999,999$ \\
\hline \hline$F_{1}, H C_{2}$ & 0.06088 & 0.05557 & 0.05465 & 0.05545 & 0.05637 \\
$F_{2}, H C_{2}$ & 0.04592 & 0.05515 & 0.05539 & 0.05516 & 0.05632 \\
\hline
\end{tabular}

Table 1: Wild bootstrap estimator

With our data, the $\mathrm{HC}_{2}$ form of the HCCME estimator is equal to 0.0562873 , the two wild bootstrap estimators are presented in Table 1 . These results make clear that, for a fixed number of observations $n=20$, the wild bootstrap estimators converge to the HCCME estimator as $B \rightarrow \infty$.

\footnotetext{
${ }^{1} x=-2.2824,-0.435864,2.27108,-1.05705,-1.10142,0.648927,0.143281,-0.25922,1.87924,-1.32969$, $0.013618,-0.303695,1.24507,0.670023,0.658823,0.521237,-0.0656568,-0.370603,-0.0734635,-0.169986$
} 


\subsection{Bias}

In this subsection, we report the bias of the wild bootstrap and HCCME estimators of $\hat{\beta}$. In our experiment, we consider the following estimators: the two wild bootstrap estimators described in the preceding subsection, the $\mathrm{HC}_{2}$ form of the HCCME, and the $\mathrm{HC}_{3}$ form of the HCCME. MacKinnon and White (1985) investigated the performance of the jackknife estimator, but subsequent computer simulations suggested that the $\mathrm{HC}_{3}$ form of the $\mathrm{HCCME}$ behaves very similarly to the jackknife, see Davidson and MacKinnon (1993, section 16.3).

We calculate the bias as the (mean) estimated variance minus the true variance of $\hat{\beta}$. In addition, we calculate the mean squared error (MSE). The number of replications is $N=9,999$ and the number of bootstrap samples is $B=499$. The results are presented in Table 2 .

\begin{tabular}{|c||cc|}
\hline Design & Bias & MSE \\
\hline \hline wboot, $F_{1}, H C_{2}$ & 0.000206 & 0.000828 \\
wboot, $F_{2}, H C_{2}$ & 0.000180 & 0.000823 \\
HCCME, $H C_{2}$ & 0.000078 & 0.000809 \\
HCCME, $H C_{3}$ & 0.013018 & 0.001645 \\
\hline
\end{tabular}

Table 2: Bias and MSE

We find the same results as Cribari-Neto and Zarkos (1999): the bias of the $H_{2}$ form of the wild bootstrap estimator is smaller than that of the $H C_{3}$ form of the HCCME. Furthermore, these results make clear that the same forms of the wild bootstrap and of the HCCME estimators have similar finite sample behavior, and that the wild bootstrap estimators (which include experimental error) are substantially worse, as theory suggests.

\subsection{Size distortion}

In this subsection we report the size distortion of tests based on the $\mathrm{HC}_{2}$ and $\mathrm{HC}_{3}$ forms of the HCCME of $\hat{\beta}$. We do not study the wild bootstrap estimator because we have shown that its finite sample behavior is quite similar to that of the HCCME. We test the null hypothesis $H_{0}: \beta=0$ with the squared $t$-statistic, which is asymptotically $\chi^{2}(1): \tau=t^{2}=[\hat{\beta} / \hat{S}(\hat{\beta})]^{2}$, where $\hat{\beta}$ is the ordinary least square parameter estimate and $\hat{S}(\hat{\beta})^{2}$ the second diagonal element of the covariance matrix estimator. The realizations of the statistics $\hat{\tau}$ are used to calculate a $P$-value at the nominal level $\alpha=0.05$. The number of replications is $N=9,999$ and the number of bootstrap samples is $B=499$.

\begin{tabular}{|c||c|}
\hline estimator & Empirical level \\
\hline \hline $\mathrm{HCCME}, H C_{2}$ & 0.0774 \\
$\mathrm{HCCME}, H C_{3}$ & 0.0547 \\
\hline
\end{tabular}

Table 3: Empirical level $(\alpha=0.05)$

Table 3 presents the empirical level of the $t$-statistics based on the $H C_{2}$ and $H C_{3}$ forms of the HCCME estimators. Tests based on the $\mathrm{HC}_{2}$ estimator reject the null hypothesis 
more than $5 \%$ of the time when it is true $(7.74 \%)$, whereas tests based on the $H C_{3}$ reject near 5\%. We find here the same results as those of MacKinnon and White (1985): tests based on the jackknife estimator, or $H_{3}$, are more reliable than others.

\section{Conclusion}

In this paper, we show that the wild bootstrap is not useful to estimate a heteroskedasticity consistent covariance matrix. However, it is well known that the bootstrap yields better results when applied to asymptotic pivots, and it is therefore a better idea to use HCCME in order to construct such asymptotic pivots before using the bootstrap, see Horowitz (2000) for an account of the relevant issues.

\section{References}

Chesher, A. and I. Jewitt (1987). "The bias of a heteroskedasticity consistent covariance matrix estimator". Econometrica 55, 1217-1222.

Cribari-Neto, F. and S. G. Zarkos (1999). "Bootstrap methods for heteroskedastic regression models: evidence on estimation and testing". Econometric Reviews 18, 211-228.

Davidson, R. and J. G. MacKinnon (1993). Estimation and Inference in Econometrics. New York: Oxford University Press.

Eicker, B. (1963). "Limit theorems for regression with unequal and dependant errors". Annals of Mathematical statistics 34, 447-456.

Horowitz, J. L. (2000). "The bootstrap". In Handbook of Econometrics, Volume 5. J. J. Heckman and E. E. Leamer (eds), Elsevier Science.

Liu, R. Y. (1988). "Bootstrap procedure under some non-i.i.d. models". Annals of Statistics 16, 1696-1708.

MacKinnon, J. G. and H. L. White (1985). "Some heteroskedasticity consistent covariance matrix estimators with improved finite sample properties". Journal of Econometrics 21, $53-70$.

White, H. (1980). "A heteroskedasticity-consistent covariance matrix estimator and a direct test for heteroskedasticity". Econometrica 48, 817-838. 